\title{
Un choix méthodologique pour l'enseignement de la langue française en Espagne dans la première moitié du $\mathrm{XX}^{\mathrm{e}}$ siècle
}

María Inmaculada Rius Dalmau

\section{(2) OpenEdition Journals}

Édition électronique

URL : https://journals.openedition.org/dhfles/1103

DOI : 10.4000/dhfles. 1103

ISSN : 2221-4038

Éditeur

Société Internationale pour l'Histoire du Français Langue Étrangère ou Seconde

Édition imprimée

Date de publication : 1 décembre 2005

Pagination : 103-122

ISSN : 0992-7654

\section{Référence électronique}

María Inmaculada Rius Dalmau, « Un choix méthodologique pour l'enseignement de la langue française en Espagne dans la première moitié du XXe siècle ", Documents pour l'histoire du français langue étrangère ou seconde [En ligne], 35 | 2005, mis en ligne le 20 novembre 2014, consulté le 27 mai 2021. URL : http://journals.openedition.org/dhfles/1103; DOI : https://doi.org/10.4000/dhfles.1103

Ce document a été généré automatiquement le 27 mai 2021.

(c) SIHFLES 


\title{
Un choix méthodologique pour l'enseignement de la langue française en Espagne dans la première moitié $\mathrm{du} \mathrm{XX}^{\mathrm{e}}$ siècle
}

\author{
María Inmaculada Rius Dalmau
}

1 Nous présentons le choix méthodologique de Joan Nogués i Aragonés, professeur de français qui obtint sa chaire de français au lycée d'Huesca en 1927, puis travailla, de 1932 à 1939, comme professeur de français à l'Institut-Escola de Barcelone. Après la Guerre civile il se chargea de cet enseignement au lycée La Rábida à Huelva, et ensuite dans un lycée de Salamanque, jusqu'en 1971.

2 Tout d'abord, et afin de pouvoir juger de l'importance de la méthode de Joan Nogués en la situant dans son contexte, nous présenterons une vue générale de la situation de l'enseignement des langues vivantes en Espagne dans cette période.

3 Pendant le dernier tiers du XIX ${ }^{e}$ siècle, l'enseignement des langues modernes en Espagne dans les établissements officiels, était dans une situation vraiment précaire. Même si à partir de 1857 - grâce à la promulgation des premières dispositions officielles qui établissaient le caractère obligatoire de l'enseignement des langues vivantes en Espagne, connue comme loi Moyano - on trouvait l'enseignement du français dans le curriculum obligatoire de l'enseignement secondaire ${ }^{1}$, les professeurs chargés de cette tâche n'avaient pas la préparation adéquate, ni les moyens pour l'enseignement, et les conditions générales de scolarisation ne permettaient pas d'obtenir des résultats acceptables.

4 La situation au début du $\mathrm{XX}^{\mathrm{e}}$ siècle n'avait pas trop changé, au moins dans les établissements appartenant à l'État. De leur côté, les institutions éducatives privées s'occupaient mieux de l'enseignement des langues modernes ${ }^{2}$. D'une part, les congrégations religieuses, surtout celles provenant de France - les Sœurs de l'Enfant Jésus, les Frères des Écoles Chrétiennes, les Frères Maristes - concédaient à l'apprentissage de la langue française un rôle très important dans leurs plans d'étude. 
On peut dire que leurs élèves étaient habituellement en contact avec la langue et la culture françaises. D'autre part, les institutions privées d'origine laïque, par exemple les écoles de langues, les établissements dépendant des gouvernements régionaux ou les établissements de l'Institucion Libre de Enseñanza, considéraient l'enseignement des langues modernes comme un bien nécessaire qu'il fallait développer. Parallèlement, des institutions d'origine française comme l'Alliance Française, l'Institut Français, le Lycée français, l'École de la colonie française de Barcelone ou bien l'Escuela Internacional de Idiomas s'efforçaient d'offrir à leurs élèves un enseignement approprié de la langue française.

D'ailleurs, les méthodes pour l'enseignement du français employées à l'époque en Espagne dans la plupart des établissements officiels étaient sous l'influence de la méthode traditionnelle - méthode grammaticale ou méthode grammaire-traduction c'est-à-dire, la même méthode que celle employée pour l'enseignement des langues classiques. Il s'agissait d'un enseignement consacré à la description grammaticale, partant d'une conception déductive et ayant pour but la lecture dans la langue acquise et sa traduction. On peut résumer les principaux traits de cette méthode de la façon suivante :

1. Vue normative et descriptive de la langue.

2. Importance de la mémorisation de règles grammaticales et de listes de vocabulaire.

3. Étude de la syntaxe et de la morphologie hors contexte.

4. Étude du vocabulaire en relation avec les règles grammaticales mais sans envisager la communication.

5. Méthode axée sur le professeur.

6. Manque d'interaction orale dans la langue apprise.

7. Apprentissage fait d'une façon déductive.

8. Emploi habituel de la traduction (version ou thème).

9. Recours fréquent aux modèles littéraires.

10. Prononciation négligée.

6 Par contre, en général, dans les établissements éducatifs privés on trouvait souvent des exemples où le choix méthodologique pour l'enseignement des langues modernes visait plutôt un objectif essentiellement communicatif, ce qui comportait l'adoption d'approches méthodologiques qui s'éloignaient des directives de cette méthode traditionnelle. On peut affirmer que, même si elles arrivaient en retard, les nouvelles tendances méthodologiques, provenant de la réforme européenne surgie en Allemagne dans le dernier tiers du XIX siècle, avaient produit des changements en ce qui concerne l'enseignement du français dans ce type d'établissements.

7 Les traits méthodologiques répandus par la réforme européenne, en réalité ceux de la méthode directe, étaient marqués par les prémisses suivantes :

1. Pendant les cours on ne parle que la langue qu'on est en train d'apprendre.

2. Il faut apprendre la langue usuelle, d'abord la langue orale puis plus tard la langue écrite.

3. L'apprentissage se fait à partir de textes choisis autour d'une action ou d'une situation concrète.

4. La grammaire est enseignée d'une façon inductive à partir de la pratique.

5. On n'enseigne que le vocabulaire et les phrases appartenant à la vie quotidienne. Les mots nouveaux s'introduisent à l'aide de la présentation d'objets, de dessins ou par association d'idées. 
6. On doit arriver au sens des mots à l'aide du contexte mais jamais par la traduction.

7. On apprend à parler à travers les questions-réponses entre professeur et élève.

8. La prononciation doit être enseignée en suivant les directives de la science phonétique.

9. Il faut graduer l'apprentissage selon des critères de difficulté.

10. La méthode est axée sur l'élève.

est clair que quand nous parlons de nouvelles tendances méthodologiques en Espagne durant cette période, nous ne parlons pas de l'adoption de la méthode directe d'une façon stricte. Loin de cela, ce qu'on trouve normalement est l'emploi des manuels de français qui adoptent une position appartenant à la méthode grammaticale et pratique $^{3}$. On assiste aussi, surtout dans des établissements privés, au choix de la méthode mixte ou éclectique. Cette méthode adopte des éléments procédant de la méthode directe pour les relier à d'autres employés dans la méthode traditionnelle.

En relation avec les tendances qu'il y avait en Europe dans le premier tiers de $\mathrm{XX}^{\mathrm{e}}$ siècle, nous pouvons avouer que même en Allemagne ou en France, pays qui ont lancé la méthode directe, celle-ci avait très tôt présenté des difficultés d'application. Selon Christian Puren, les problèmes rencontrés par la méthode directe tiennent à deux sortes de facteurs: des facteurs externes - les conditions de scolarisation, l'hétérogénéité des groupes, le nombre d'élèves par groupe, la préparation du corps professoral - et des facteurs internes - les propres insuffisances de ses théories de référence (Puren, 1988 : 191-203).

En reprenant l'histoire de notre professeur de français, Joan Nogués, on constate que la formation qu'il a reçue dans l'Institución Libre de Enseñanza (désormais ILE) a été décisive dans sa vie professionnelle. L'ILE est née en 1876 comme une alternative à l'éducation officielle espagnole toujours marquée par l'imposition du dogme catholique. La nouvelle institution éducative proposait un enseignement fondé sur des principes de liberté et de tolérance. Le krausisme, introduit en Espagne par Julián Sanz del Río, avait marqué les fondateurs de l'ILE, surtout son principal représentant, Francisco Giner de los Ríos. Pour les responsables de l'ILE, l'éducation jouait un rôle très important dans la société puisqu'elle devenait le moteur de la transformation sociale et de la modernisation de l'Espagne. La philosophie de Karl Friedrich Krause, unie à la nouvelle pédagogie européenne provenant de Rousseau, Pestalozzi ou Fröbel amenait les penseurs de l'Institución à considérer l'homme comme le centre de l'éducation. Sous cette vision pédagogique, l'élève devait apprendre à partir de son activité personnelle et de sa propre expérience, et le professeur avait pour responsabilité de stimuler, orienter l'élève et de lui fournir les moyens nécessaires. C'était la fin de l'enseignement magistral et le début de l'enseignement actif.

11 On considère l'ILE comme un prédécesseur de l'École Nouvelle en Europe. En même temps, en ce qui concerne l'enseignement des langues modernes en Espagne, nous pouvons affirmer que l'Institución a développé fortement sa présence dans les plans d'études (cf. Rius 2006). Le désir d'ouverture et de modernisation est souvent lié au besoin de connaissance des langues. D'autre part, il est remarquable de noter que l'action de l'ILE a dépassé l'enseignement privé. Des gens provenant de l'Institución ont eu des responsabilités politiques - tendance qui à commencé à partir de 1881 - et ils ont $\mathrm{pu}$ faire entrer dans l'enseignement officiel espagnol un plan pilote pour l'enseignement secondaire. On a créé des centres expérimentaux appelés InstitutoEscuela. Le premier de ces établissements a été fondé à Madrid, en 1918, et plus tard à 
Barcelone et à Valence, en 1932. Dans le plan d'études de l'Instituto-Escuela la présence de l'enseignement des langues modernes était bien supérieure à celle des Institutos provinciales $^{4}$. En ce qui concerne l'Instituto-Escuela, on trouvait le français comme langue obligatoire pendant toutes les années du secondaire, et même dans les années préparatoires. D'autre part, les élèves devaient choisir une seconde langue étrangère l'anglais ou l'allemand - qui était étudiée pendant quatre années ${ }^{5}$. De même, les professeurs qui se formaient dans l'Instituto-Escuela devaient apprendre un minimum de deux langues étrangères et ils étaient aussi obligés, au long de leur formation, de faire un stage dans un pays étranger.

Nous avons déjà dit que Joan Nogués fut élève de l'ILE puis, plus tard, professeur de l'Institut-Escola de Barcelone. Nous pensons que les principes pédagogiques de l'Institución ont eu une forte influence sur la façon d'agir, professionnellement, de ce professeur de français. Nous proposons maintenant de faire une analyse de sa méthode pour l'enseignement de la langue française, en partant de deux sources d'information: les idées que lui-même nous a laissées dans deux articles publiés (cf. Nogués, 1933 ; puis Nogués, 1959) et les annotations qu'il faisait dans son cahier personnel pour la préparation de ses cours de français.

13 À travers ses articles, Nogués indique ce qu'il considère comme les parties de sa méthode : le vocabulaire et la phrase, les contes, la grammaire et la phonétique. Pour le vocabulaire et la phrase, il avoue la nécessité de présenter le vocabulaire dans un contexte dramatique. Il faut que le vocabulaire lui-même devienne une narration vivante. Le professeur est obligé de choisir des textes contenant les mots voulus ou bien d'inventer son propre texte. Nogués considère aussi, dès le premier jour, la phrase comme un élément vivant. De leur côté, les contes et les fables sont, à son avis, des instruments didactiques très importants parce qu'ils suscitent l'intérêt de l'enfant et parce que leur structure narrative se répète d'un texte à l'autre. Par ailleurs, les contes aident le professeur à vaincre la résistance que les élèves manifestent d'habitude quand il s'agit de parler. Nogués utilise aussi le dessin et la photographie pour présenter le vocabulaire et, surtout, pour faire parler l'apprenant.

Concernant la grammaire, notre professeur insiste sur l'importance de la structure verbale. Mais, afin d'éviter la monotonie de la conjugaison, il propose de conjuguer le verbe par des phrases où les compléments soient des mots déjà connus par l'élève. Nogués affirme que le reste de la grammaire, l'élève l'apprend en parlant et en lisant. Par conséquent, il adopte un point de vue inductif pour l'enseignement de la grammaire.

Enfin, Joan Nogués se sert de la chanson populaire pour enseigner la phonétique. Il montre les sons et il corrige la prononciation à l'aide des chansons.

Nous avons pu consulter un cahier utilisé par Joan Nogués pour la préparation de ses cours de français, dont nous proposons ici un extrait ${ }^{6}$.

Vocales :

$a \mathrm{i}=\mathrm{e}$

$\mathrm{ou}=\mathrm{u}$

oi $=$ ua

$\mathrm{au}-\mathrm{eau}=\mathrm{o}$

$\mathrm{eu}=\propto$

$œ u=œ$ 


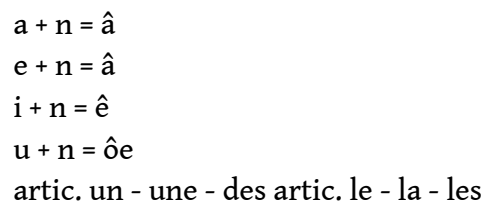

Vocabulario. Une grande salle de classe, une salle de classe longue, les pupitres, les bancs, les rangs. Combien de rangs y a-t-il ? Il y en a quatre, le tableau noir, le papier, les livres, le sous-main, le buvard, le cuir, le crayon, la plume, le porte-plume, le fauteuil du professeur, les chaises des élèves, la chaise, le poêle, le séchant [sic], le chauffage central, les radiateurs. Combien d'éléments ont les radiateurs? Ils ont six éléments. Le plafond, le plancher, l'encrier, la table, la fenêtre, la lampe, la porte, l'armoire, la règle, la serviette et la carte.

Regardez, montrez la salle de classe.

" " les pupitres, le banc, la table, les rangs.

" "le tableau noir, le papier, le livre, la plume, le crayon.

" "le sous-main, le buvard, le cuir.

" "le fauteuil du professeur, les chaises des élèves.

" "le poêle, le radiateur.

" "le plafond, le plancher, les murs, les fenêtres, la lampe.

" "la porte, le battant de la porte, la poignée de la porte.

" "l'encrier, la gomme, la boîte.

21 C'est.

22 I C'est le tableau noir, c'est la salle de classe, c'est le crayon, c'est le poêle, c'est le radiateur, c'est le mur, c'est la porte, c'est la table, c'est la serviette, c'est la lampe, c'est la fenêtre, c'est le balcon, etc.

II Qu'est-ce que c'est? C'est le livre. Qu'est-ce que c'est ? C'est la boîte. Qu'est-ce que c'est? C'est le mur. Qu'est-ce que c'est ? C'est la plume. Qu'est-ce que c'est ? C'est la lampe. Qu'est-ce que c'est? C'est le plafond. Qu'est-ce que c'est? C'est la gomme. Qu'est-ce quec'est? C'est la règle. Qu'est-ce que c'est? C'est la table. Qu'est-ce que c'est? C'est la serviette. Qu'est-ce que c'est ? C'est le papier. Qu'est-ce que c'est ? C'est la table. Qu'est-ce que c'est? C'est le banc, le tableau, etc.

III Est-ce le mur? Oui Monsieur, c'est le mur. Est-ce le pupitre? Oui Monsieur, c'est le pupitre. Est-ce le balcon? Oui Monsieur, c'est le balcon. Est-ce la chaise ? Oui Monsieur, c'est la chaise. Est-ce le fauteuil ? Oui Monsieur, c'est le fauteuil. Est-ce? Non, ce n'est pas. Est-ce l'armoire? Non Monsieur, ce n'est pas l'armoire, c'est la table. Est-ce la serviette? Non Monsieur, ce n'est pas la serviette, c'est le cartable. Est-ce le crayon? Non Monsieur, ce n'est pas le crayon, c'est la plume. Est-ce le banc? Non Monsieur, ce n'est pas le banc, c'est la table, le pupitre. Est-ce le porte-plume? Non Monsieur, ce n'est pas le porte-plume, ni le crayon, c'est la règle. Est-ce la fenêtre ? Non Monsieur, ce n'est pas la fenêtre, ce n'est ni la fenêtre ni la porte, mais la poignée de la porte. Est-ce la table ou le pupitre ? Non Monsieur, ce n'est pas la table ni le pupitre mais l'estrade.

Donnez-moi un livre, une plume, une gomme, un canif, un papier.

Voici un livre, voici un papier, voici une gomme, voici un canif, voici une plume, voici un morceau de craie.

Donnez-moi une serviette. Voici une serviette.

Donnez-moi une gomme. Voici une gomme.

Donnez-moi un sous-main. Voici un sous-main.

Donnez-moi un crayon. Voici un crayon. 
Donnez-moi votre sac à main. Voici mon sac à main.

Donnez-moi votre cache-col. Voici mon cache-col.

IV Où est la porte? Où est la fenêtre? Où est la table ? Où est la classe ? Où est le professeur? Où est le garçon? Où est le cahier? Où est l'encrier? Où est le tableau ? Où est la craie?

Où est la porte? La porte est dans la classe. Où est la fenêtre ? La fenêtre est à droite de la salle de classe. Où est la table ? La table est contre le mur. Où est la classe ? Elle est au premier étage. Où est le professeur? Le professeur est devant ses élèves. Où est le garçon? Le garçon est dans la classe. Où est le cahier? Le cahier est sur la table. Où est l'encrier ? L'encrier est sur la table. Où est l'encre ? L'encre est dans l'encrier.

Comment? adjetivos, nombres de color.

Comment est le papier? (le papier est blanc) Comment est la classe? La classe est grande. Comment est l'élève? L'élève est jeune. Comment est le professeur? Le professeur est âgé. Comment est le banc ? Le banc est dur. Comment est la fenêtre ? La fenêtre est assez grande. Comment est le mur? Le mur est blanc. Comment est la lampe ? La lampe est assez grande et brillante. Comment est le fauteuil ? Le fauteuil est mal [sic]. Comment est le porte-plume ? Le porte-plume est en bois, en métal, en or, en argent, [...], en celluloïd, etc. Comment est Mademoiselle Marie ? Mademoiselle Marie est brune, mais Mademoiselle Marguerite est blonde. Comment est la porte, petite ou grande ? Elle est grande. [...]

Les actions de la classe.

Présent d'indicatif. Impératif, formes : entre, entrons, entrez

J'entre dans la classe. Entrez dans la classe.

Je m'assieds sur ma chaise. Asseyez-vous sur la chaise.

Je prépare mon cahier. Préparez votre cahier.

Je prends mon cahier et Prenez votre cahier.

J'écris des phrases. Ouvrez le livre.

J'ouvre le livre. Fermez la porte.

Je ferme le livre. Ouvrez la porte.

Je ferme la porte. Levez-vous,s'il vous plaît.

J'ouvre la porte.

Je me lève.

Qui est-ce qui ? Qu'est-ce qui est?

Qu'est-ce qui est blanc? Le chien, le cheval sont blancs. Qu'est-ce qui est rouge ?... Le toit de la maison est rouge. Qu'est-ce qu'est le vert ? Le vert est une couleur. Qui est-ce qui est un homme? Jean est un homme. Qui est-ce qui est un garçon? Paul est un garçon. Qu'est-ce qu'est Marie? Marie est une jeune fille. Qui est-ce qui écrit? L'élève écrit. Qui est-ce qui apprend aux élèves ? Le professeur apprend aux élèves. [...] Qu'estce qui est blanc ? Le papier est blanc. Qui est-ce qui lit ? L'élève lit....

Voici, voilà, ici, là. Que faites-vous?

Où est la table ? Elle est ici. Où est l'encrier ? L'encrier est sur la table, là, le voilà. Où est l'élève ? L'élève est près de nous, le voici. Où est la plume? La voilà. Comment est le livre? Le livre est grand, amusant, le voici. Comment est l'armoire? L'armoire est 
haute, grande, la voilà. [...] Que faites-vous ? Nous entrons, nous nous asseyons, nous ouvrons nos livres, nous écrivons sur nos cahiers, nous fermons nos livres et nous nous levons. Qui est-ce qui travaille? C'est Jean qui travaille. Qu'est-ce qu'il travaille? Il travaille à ses devoirs. Où est la lampe ? Elle est suspendue du plafond. Comment est la lampe ? Elle est claire et brillante. Que fait la lampe ? Elle nous éclaire. Qu'est ce qui est vert? L'arbre est vert. Qu'est-ce qui est rouge? Le sang est rouge. Qui est-ce qui marche, s'assied, ouvre le livre? L'élève marche, ouvre le livre, etc. Que fait l'élève? L'élève vient d'entrer. Que fait Marie? Elle vient de sortir. Que fait le concierge? Il veille à l'ordre des couloirs. [...] Que fait Monsieur? Il ouvre la porte. Où est la porte? Elle est près de la table. Comment est la table? Elle est assez grande. Qu'est-ce qui est brun? Le bois de la table est brun.

Imparfait de l'indicatif d'avoir et être.

J'étais, tu étais il était, n. étions, v. étiez, ils étaient.

J'avais, tu avais, il avait, n. avions, vous aviez, ils avaient. [...]

Le vocabulaire du corps humain.

La tête, le tronc et les membres composent le corps humain.

Nous sommes en classe, les élèves sont assis, ils écrivent sur le cahier, ils lisent la leçon, ils se lèvent, ils sont debout, ils ouvrent le livre, ils ferment le livre, ils préparent leurs devoirs, ils parlent, ils se taisent. Si le professeur leur dit lisez, ils lisent, si le professeur leur commande ouvrez le livre, ils l'ouvrent. Asseyez-vous, dit le professeur, ou levezvous, alors ils se lèvent et ils s'asseyent. Asseyez-vous, c'est l'impératif d'asseoir.

Nous avons fait des phrases qui commencent par comment, où, voici, voilà, Est-ce que. Qu'est-ce qui est. Qui est-ce qui est, combien, etc.

Aujourd'hui nous allons parler du vocabulaire du corps humain.

La partie la plus intéressante de l'homme est la tête. Il y a des têtes grandes et des têtes petites. Le singe a une petite tête. L'éléphant a une grosse tête. L'enfant a des cheveux noirs, la petite fille a les cheveux blonds. Mademoiselle Thérèse a les cheveux châtains. Louise a les cheveux courts et frisés. Toinette a les cheveux longs et bouclés, le nègre et la négresse [sic] ont les cheveux crépus. Le front des enfants est charmant, le front des vieillards est ridé. Les yeux sont bleus, noirs, châtains, verts, gris, cela dépend. Le cou est long ou court, le taureau a le cou court et robuste, le héron a le cou long emmanché à un long bec. Le col de la chemise est commode s'il est large ou pas commode s'il est étroit. Les épaules peuvent être aussi larges ou étroites. Le dos de l'homme se tient par la colonne vertébrale. La poitrine est relevée par l'effort des poumons. Les bras, les cuisses et les jambes, avec les pieds sont d'autres parties plus petites du corps humain.

Combien de bras avez-vous? Combien d'oreilles? Combien d'yeux? Combien de bouches? Combien de cerveaux avons-nous? Combien de dents? Combien de poumons, de jambes, de cuisses, de pieds, de doigts?

A quoi sert la tête, les oreilles, le nez, les yeux, la langue, la peau?

Les oreilles servent à sentir.

Où sont les oreilles, la tête, la bouche, les dents, la langue?

Le corps humain

Nous avons parlé de la tête, des cheveux, de la bouche, des joues, des yeux.

Les oreilles du musicien sont grandes, les oreilles de la souris sont rondes, les oreilles de l'éléphant sont pendantes. A quoi servent les oreilles? A entendre. Combien 
d'oreilles avez-vous ? Le menton est le bout de la barbe ; il y a des mâchoires robustes et d'autres fragiles. Votre mâchoire, comment est-elle?

Le col de la chemise, le col de la montagne [...] Le cou attache la tête au tronc. Qu'est-ce que c'est le menton? Les mâchoires sont toujours du même type ? Où est contenu le cœur, le poumon? Que contient la poitrine ? [...]

A quoi servent les bras? Les bras servent à embrasser, étreindre, à lever des poids, à travailler, etc. Où sont situés les bras? Ils se rattachent aux épaules et ils sont situés symétriquement des deux côtés du tronc, et la main, à quoi sert-elle? La main est encore plus utile que le bras car on s'en sert pour saisir, prendre, toucher, écrire, s'habiller, se laver, se raser, se coiffer, etc. Que ferions-nous sans les mains ? La vie des mutilés de guerre est pénible. Son [sic] adaptation a créé des problèmes qui n'existaient [sic] avant la guerre. Presque tous les métiers font un emploi exclusif des mains; le professeur les emploie pour écrire, le chauffeur pour guider le volant, le boulanger pour pétrir la farine, [...] le forgeron frappe le fer tandis qu'il est chaud, mais que feraitil sans les mains? [...] Le dos de la main a les veines superficielles tandis que la paume, plus lisse malgré ses rides, ne les a pas.

Nous allons visiter la famille Fernández (Durand)

Nous voici devant sa maison. Voyez-vous sa maison? Pouvez-vous dire comment est sa maison? Petite, grande, [elle] a plusieurs étages? Combien de balcons y voyez-vous? Le toit est-il rouge ? Y a-t-il deux ou quatre fenêtres à la façade ? La porte d'entrée a devant elle un escalier? Voyez-vous le balcon? Voyez-vous le rez-de-chaussée et le garage? Où est le grenier? Où est la cave ? Nous montons par l'escalier, il a quatre marches. On pousse le bouton [sic], on sonne. Nous attendons quelques secondes, pas minutes. On ouvre la porte, la bonne est devant la porte. Monsieur et Mme Fernández sont-ils à la maison? Oui Messieurs, entrez s'il vous plaît. [Description des membres de la famille et présentation des mots : parents, enfants, fils, fille, épouse, mariée, père, mère, grands-parents, sœur, frère, tante, oncle, cousin, beau-frère, belle-sœur, ...]

Nous saluons les maîtres de la maison :

- Comment allez-vous Mme. Fernández ? C'est un plaisir de vous saluer. mes a mes anis. Margueritte Delpi, Catherine Dieulafait, Armande Bejast, M. Gaston, mon beau-frère, ma tante Denise, mon mari Pierre, mes enfants, Marie, l'aînée, Charles, le cadet, et si vous voulez aller saluer mes grands-parents, ils sont là, près de la cheminée. [...]

La salle à manger

Nous passons à la salle à manger. Quelle heure est-il ? Et pourquoi demandons-nous quelle heure est-il ? Y a-t-il de [sic] rapport entre la salle à manger et l'heure ? Est-ce que nous sommes à tous les moments dans la salle à manger? Non, nous sommes à huit heures, parfois à neuf heures dans la salle à manger. Qu'est-ce qu'on fait à huit heures dans la salle à manger ? On y prend le petit déjeuner. Et à midi, que faisons-nous dans la même pièce? On y déjeune, on y prend le déjeuner. Et à sept, huit ou neuf heures, pourquoi sommes-nous réunis dans la salle à manger? On en comprend la raison parfaitement : Voilà expliqués les différents moments dans lesquels nous sommes dans la salle à manger. [Description de la salle à manger et de ce que fait Marie, la bonne de la famille, pour préparer la salle avant le repas. Présentation des mots : nappe, assiette, cuillère, couteau, fourchette, serviette, verre, etc.] 
66 - Avez-vous faim, mes amis? dit Mme. Durand à ses fils. Oui maman, nous avons de l'appétit, beaucoup d'appétit. Alors, c'est le moment de s'attabler, dit Mme. Durand. D'abord Marie apporte une grande soupière. Qu'y a-t-il dedans? Il ne faut pas le demander, la soupe fumante fume. Parfois ce n'est pas la soupe à la vermicelle [sic] dont le bouillon est agréable, mais un potage qui fume aussi, ce qui est apporté par la bonne. Les macaroni [sic], les légumes, le riz, le pot à feu, sont servis à table au déjeuner chez Mme. Durand. Les enfants boivent de l'eau, les parents de la bière ou du vin. Je veux du vin, s'écrie Charlot le petit de la maison. Les enfants ne boivent pas du vin [sic], reprend M. Durand d'un ton irrité. - Je veux boire de la bière, dit le second, maman elle en boit. - Oui, mon enfant, mais ce n'est pas bon pour la santé des enfants de la boire si on est petit comme toi, répond la mère. [...]

67 - Qu'est-ce que tu voulais dire il y a un moment?

68 - Ce n'est pas la peine maintenant.

69 - Pourquoi?

70 - Tu avais une chenille dans ta salade, maintenant tu l'as avalée.

71 M. Durand ressent quelque chose de désagréable à l'estomac. La famille mange en silence.

72 - Gaston, dit la mère, ramasse ta serviette, elle est tombée.

73 - Oui maman.

74 - Tu n'aimes pas la soupe à la vermicelle [sic]?

75 - Non maman.

76 - Pourtant elle est bonne! Tu n'aimes pas la soupe aux choux, tu n'aimes pas la soupe aux fines herbes, tu n'aimes pas non plus le potage, tu n'aimes pas le pot à feu, alors, qu'est-ce que tu aimes?

77 - Je n'aime pas la soupe.

78 - Et le ragoût, l'aimes-tu? Et la viande ? Et le poisson?

79 - Il n'aime rien, n'importe quoi que se soit, dit la petite Suzette. Et puis, il torche ses mains dans les pantalons quand personne ne le regarde. Il est rachitique.

80 - Tu es une sale espionne, dit le petit Charlot roulant des yeux.

81 - Paix, dit M. Durand, d'un ton courroucé. Ces enfants n'ont pas de l'appétit [sic]. Il faudra leur faire quelques piqûres. Les enfants doivent manger toute sorte d'aliments : des légumes, des haricots verts, des choux, de la salade, des radis, de la viande, du poisson, le dessert, etc.

82 - Ce que nous aimons le plus sont les etc. dit Gaston : le dessert, les fruits, les gâteaux.

83 Les différentes pièces de l'appartement. [L'auteur en fait une description détaillée en faisant un parcours par les différentes pièces].

84 [...] Nous sommes à la cuisine : ce qui frappe notre attention c'est la cuisinière, le fourneau, l'armoire, l'évier et les différents utils [sic] qui sont employés dans cette pièce. Que fait la cuisinière ou la ménagère dans la cuisine? Et bien, elle cuisine. La salle de bain vous intéresse-t-elle? Voulez-vous prendre un bain ? On en parlera. Et le garde-manger, vous intéresse-t-il ? Que peut-on y faire, dans un garde-manger ? On n'y fait rien du tout. On y entasse les aliments, les fruits, les conserves. N'oubliez pas que 
nous conservons les aliments qui se gâtent facilement dans le frigidaire. Les caves paysannes servent aussi pour y mettre certaines denrées, les jambons, les cervelas.

Nous allons passer revue à la maison [sic]. Elle a un hall, un couloir, une cuisine, une salle de bains, une salle à manger, un salon, un cabinet de travail, et différentes salles à coucher pour les différents membres de la famille. Que fait-on au hall, au vestibule ? On attend le moment où le maître de la maison va nous recevoir. Que fait-on au couloir ? On n'y fait rien, généralement on y marche pour arriver à des pièces déterminées.

Il est huit heures du matin. Le réveille à matin sonne. Maman a mis quelques pièces de monnaie à côté du réveille-matin et la table de toilette résonne, retentit, elle fait un bruit épouvantable, strident. Charlot doit se préparer à se réveiller, voilà, il n'y a plus de sommeil pour le pauvre Charlot. Il faut se décider, - à quoi ? - À rien du tout. Le linge est si chaud, les couvertures si chaudes, l'édredon si mou.

- Charlot, lève-toi! Tu vas à arriver tard au lycée [sic]. Dépêche-toi, mon cher. N'attends que j'aille te faire sauter du lit. Maman! Ah! Maman! Qu'elle est dure, cruelle, incapable de comprendre les enfants comme Charlot. Est-ce que ne va-t-il pas [sic] se pouvoir dégourdir un peu, s'étirer... ? Est-ce que la paresse est quelque chose qui ne mérite pas de ménagements? Au bout de quelques minutes Papa entre dans la chambre à coucher de Charlot. Il enlève les couvertures et voilà le pauvre Charlot en pyjama sur le lit. Voyons, tu es idiot! Tu vas arriver tard au lycée! Et Charlot se voit debout sur la descente du lit et tandis qu'il tâche de se mettre les chaussettes Papa - ah, qu'il est abominable ce qu'on fait avec le pauvre Charlot ! Papa l'empoigne et le fait courir par le couloir froid, brrr ! Et arriver à la salle de bains. [...] la voix rude de Papa sonne dans ses oreilles : Lave-toi ! Se laver ? Est-ce qu'on peut se laver avec de l'eau froide comme ça? Mais Papa dit Bon, je vais te laver moi-même, et il baisse la tête de Charlot au dessous du robinet à l'eau chaude - qui n'est pas chaude - et l'eau froide jaillit avec violence. C'est comme une tape d'eau froide, une gifle sur la nuque. Au bout d'un moment Charlot s'apaise et comprend que le monstre froid du robinet a été vaurien [sic]. Dans sa chambre, il met ses chaussettes, son caleçon, sa chemisette, sa chemise, il passe autour de son cou sa cravate, il boutonne [...] il met son chandail chaud de laine, son veston d'hiver de gros drap de laine; son cache-col et presque content va à la salle à manger pour prendre son petit-déjeuner. D'abord il enduit soigneusement deux grosses tranches de pain avec du beurre. Boit son café au lait. Puis il mange de la marmelade et avec sa serviette se dirige au lycée. [...]

Les heures et le temps.

Nous avons déjà parlé du réveille-matin; mais nous n'avons pas parlé des autres montres et de l'horloge. Il y a des montres bracelets qui s'attachent au poignet. Elles sont en acier, en argent, en nickel ou en or. La montre a un cadran et une grande aiguille qui marque les heures et une petite aiguille qui marque les secondes sur un cercle. On remonte une montre à l'aide du remontoir. Le jour a vingt-quatre heures. L'horloge de la cathédrale marque 4 heures de l'après-midi. La pendule de la salle à manger avance de cinq minutes. La montre de M. Durand est en retard. Elle retarde d'une minute par jour. Quelle heure est-il ? Il est huit heures sonnées. Il est huit heures et quart. L'horloge de la cathédrale sonne les heures, les demies et les quarts. A midi le soleil brille s'il n'y a pas de nuages. Le matin à Salamanque est froid, l'après-midi (de midi à six heures) le soleil s'en va, le soleil se couche en hiver. Le soir (de six heures à minuit) les ombres du soir se répandent sur Salamanque. Si vous saluez une personne le matin, vous lui dites bonjour M. ! Bonjour Mme! Vous êtes arrivée en avance, vous êtes 
arrivée en retard; si c'est l'après-midi on dit bon soir et au moment de se coucher on dit bonne nuit. A neuf heures sonnant nous sortons de notre cours, une horloge sonnant répète les heures.

Que faites-vous à huit heures du matin ? C'est difficile à répondre car il y a des jeunes gens qui dorment, d'autres qui sommeillent, d'autres qui se lèvent, d'autres qui se sont levés, d'autres qui sont en retard ou en avance, et il y a aussi ceux qui se lavent, qui déjeunent ou qui sont à leur travail. A quelle heure entrez-vous dans vos premières classes? Vous entrez à 9 heures sonnant mais si le professeur est en retard vous entrez à neuf heures dix minutes, et parfois si le professeur n'est pas ponctuel, exact, régulier, vous entrez à neuf heures et quart. Que faites-vous à dix heures ? ...

Le jour a 24 heures. L'heure 60 minutes. La minute 60 ... Une semaine a.... Un mois a 30, 31, 29 ou 28 jours. Les divisions du mois sont le semestre, le quadrimestre, etc. La veille de Noël en Espagne c'est la Noche Buena. Le lendemain c'est Noël. L'avenir c'est le temps du futur, celui qui n'est pas encore. Le passé c'est le temps écoulé.

Les spectacles

Quels sont les spectacles de la ville?

Le premier spectacle est celui des rues de la ville. Voilà pourquoi il y a des postes de signalisation avec des lumières qui indiquent le moment où l'on peut passer et le moment d'arrêt, d'attente, etc.

Puis il y a les agents de ville qui marquent avec des sifflets ou avec les mains les moments où l'on doit passer ou dans lequel endroit [sic] s'arrêter.

La rue dite la Rua, n'a pas beaucoup de mouvement; elle est étroite, ou assez étroite. Elle est une vieille rue de Salamanque. Elle a des établissements commerciaux où l'on vend. [...] A juger par les devantures nous voyons une horlogerie à la devanture de laquelle on voit des montres bracelets, des lunettes de toutes sortes, grandeurs et proportions. En face il y a une librairie ; dans cette librairie il y a des volumes de livres de matières différentes; ceux qui abondent sont les romans, et dans ce genre littéraire il y a des romans sérieux, des romans roses ou blancs pour la femme, des livres religieux, de propagande religieuse, etc. Mais ce qui abonde le plus sur les devantures des librairies sont les romans étrangers, des traductions de romans anglais et américains, des romans policiers. Les livres traduits sont des gros volumes reliés avec des couvertures en couleurs voyantes qui frappent la vue des passants. Comme il arrive souvent après les guerres, les romans qui sont les représentants de l'évasion de l'individu dans la société se développent énormément et dans les temps modernes il faudra fixer l'attention en ce fait [sic].

En face de l'horlogerie il y a un orfèvre; c'est une devanture petite qui a les vitres aux deux donnants [sic] de la rue et de la place. On peut y voir des verres et des coupes taillées et avec des ornements en argent ; des [sic] polis bijoux tels que des pendentifs où l'on a gravé des [sic] jolies figures, [...] des bracelets, des bagues, des agrafes, des aiguilles, des petites boîtes en argent dont les ornements sont ceux de la Renaissance. [...] Il n'y a pas d'accord dans la dénomination des établissements français et espagnols. Le vocabulaire français manque des noms tels que paragüería. Traduit moyennant la périphrase marchand de parapluies, marchand de chaises (sillería), marchand de vaisselle (cacharrería) ou bien elle emploie chez le coiffeur (peluquería), chez le 
cordonnier (zapatería), chez la modiste (sombrerería de señoras), chez le tapissier, chez le serrurier, chez le vitrier, chez le charpentier, chez le menuisier, etc.

Nous pouvons constater tout d'abord que Joan Nogués utilise la technique questionréponse impulsée par la méthode directe. Il fait répondre à ses élèves négativement et affirmativement et il complique progressivement les structures syntaxiques. Nous repérons aussi que souvent le professeur introduit une nouvelle leçon en faisant référence à des notions déjà travaillées avant - par exemple, avant de commencer le vocabulaire du corps humain. Également il fait des récapitulations à la fin d'une leçon par exemple, à la fin de l'explication des différentes pièces de l'appartement.

Est très intéressante, à notre avis, la richesse de nuances que Nogués met dans ses présentations de vocabulaire et dans les situations qu'il crée. Au sein des textes de son cahier, tout est fortement lié à la vie réelle. Son effort pour atteindre les élèves devient évident quand il adopte leur point de vue et quand il se rapproche d'eux avec un naturel extraordinaire. Parfois, il se produit une sorte de complicité entre le texte et l'élève. Par exemple quand Nogués décrit le début de la journée, moment de se réveiller pour aller au lycée. Les mots ne sont jamais isolés. On aperçoit un sens général qui relie tout l'ensemble.

De son côté, notre professeur veille bien à introduire dans ses textes des réflexions et des contenus qui visent une formation générale de l'individu. Par exemple, quand il parle du corps humain, concrètement de la main, il nous rappelle: "les ongles propres ». Dans ce même contexte il réfléchit aussi sur la vie des mutilés de guerre. En lisant le cahier de Nogués on trouve de nombreux exemples de cette sorte de réflexions : pendant la visite à la famille Fernández-Durand, au moment de visiter la librairie de la ville, etc.

À notre avis, les traits les plus significatifs de la méthode de Joan Nogués sont les suivants :

1. Lire, parler et traduire sont des buts de l'enseignement d'une langue étrangère.

2. La motivation des élèves est une tâche fondamentale du professeur.

3. Le dessin constitue la représentation graphique du vocabulaire.

4. Le professeur doit surveiller la prononciation correcte de ses élèves.

5. Le principe de la méthode active ne peut pas être discuté.

6. Les activités didactiques doivent être présentées d'une façon simple et claire.

7. Le vocabulaire doit se présenter dans un ensemble narratif afin qu'il devienne narration vivante.

8. La maitrise syntaxique d'une centaine de mots est préférable à la connaissance par cœur de nombreux vocabulaires.

9. Le vocabulaire oblige à présenter des textes littéraires qui en soient un complément.

10. On doit avoir soin des intérêts des élèves en choisissant ou élaborant des textes qui soient appropriés à leur âge et à leurs attentes.

11. Il faut prévoir un choix de textes dont le contenu humain vise la formation générale de l'individu.

12. La connaissance de la structure verbale est indispensable dans l'apprentissage d'une langue.

13. Il est important de faire une gradation progressive des difficultés.

14. On doit intégrer les connaissances déjà acquises par les élèves dans la présentation de nouveaux concepts et règles de la langue. 
Nous pouvons conclure que Joan Nogués constitue un bon exemple de ce que l'action de l'Institución Libre de Enseñanza a impulsé en relation avec l'enseignement des langues modernes en Espagne. Cela se matérialise par l'adoption des principes de la méthode active - dans une perspective pédagogique générale - et par l'adoption aussi d'instruments novateurs pour l'enseignement des langues modernes. On peut situer Nogués dans la même tendance que celle qu'il y avait en Europe à son époque, c'est-àdire, l'emploi d'une méthode mixte marquée par l'éclecticisme. L'adoption des principes de la méthode directe pour le premier contact avec la nouvelle langue, la présentation du vocabulaire en contexte, l'enseignement de la grammaire d'une façon inductive, la stimulation de l'activité de l'élève, la motivation à l'égard de l'apprentissage, l'insistance sur la prononciation, la formation culturelle et littéraire, et finalement, la traduction de textes littéraires pour les élèves du niveau avancé, sont des traits qui caractérisent le choix méthodologique de ce professeur et qui montrent sa modernité, surtout par rapport à ce qu'on trouvait dans la plupart des établissements scolaires officiels espagnols à ce moment-là.

\section{BIBLIOGRAPHIE}

BOUDES, Odette. 1925. « Enseñanza de las leguas modernas ». In Un ensayo pedagógico. El InstitutoEscuela de segunda enseñanza de Madrid. Madrid : Junta Para la Ampliación de Estudios e Investigaciones Científicas.

CAPITÁN DÍAZ, Alonso. 2002. Breve historia de la educación en España. Madrid : Alianza Editorial.

GARCÍA-BASCUÑANA, Juan. 1999. « L'institutionnalisation du FLE dans l'enseignement public espagnol après la Loi Moyano (1857) : avatars et conséquences ». Documents 23 : 108-123.

GÓMEZ MOLLEDA, Mํㅡㄹ Dolores. 1966. Los reformadores de la enseñanza contemporánea.Madrid : Escuela de Historia Moderna.

INSTITUT-ESCOLA. 1992. Institut-Escola, 1932-1939. Replega d'escrits amb motiu del $60^{\grave{e}}$ aniversari de la creació de l'Institut-Escola. Barcelona : Generalitat de Catalunya, Departament de Cultura. JIMÉNEZ-LANDI, Antonio. 1996. La Institución Libre de Enseñanza y su ambiente.Madrid : Editorial Complutense.

JUNTA PARA LA AMPLIACIÓN DE ESTUDIOS E INVESTIGACIONES CIENTÍFICAS. 1925. Un ensayo pedagógico. El Instituto-Escuela de Segunda Enseñanza de Madrid. (Organización, Métodos y Resultados). Madrid.

NOGUÉS ARAGONÉS, Joan. 1933. « El francès a l'Institut-Escola ». Bulletí de L'Institut-Escola 13 : 4-6. NOGUÉS ARAGONÉS, Joan. 1959. «Renovación del libro de texto », Publicaciones de la revista de Enseñanza Media 261, Nuevos Métodos en la Enseñanza de Idiomas. Madrid : Ministerio de Educación Nacional, Colección Guías Didácticas, 73-78.

PUREN, Christian. 1988. Histoire des méthodologies de l'enseignement des langues.Paris : CLE International. 
ROIG, Carmen. 1990. «Le français dans les programmes officiels en Espagne : 1934-1938 ».

Documents $6: 212-234$.

ROIG, Carmen. 1997. « L'enseignement du FLE au sein de l'Institución Libre de Enseñanza dans le premier tiers du XXe siècle ». Documents 20 : 141-157.

RIUS DALMAU, M Immaculada. 2006. La enseñanza del francés en el marco de la Institución Libre de enseñanza (1876-1939). Tarragona : Universitat Rovira i Virgili [thèse de doctorat inédite].

SÁNCHEZ, Aquilino. 1997. Los métodos en la enseñanza de idiomas. Evolución histórica y análisis didáctico. Madrid : SGEL.

\section{NOTES}

1. Presque la seule langue étrangère enseignée était le français, surtout dans les Études Générales. Dans les Études d'Applicationon trouvait aussi la présence de l'allemand et l'anglais.

2. À cette époque, et jusqu'en 1933 - année de l'approbation par le gouvernement de la IIe République de la loi des Congrégations religieuses qui supposait la suppression des activités éducatives de celles-ci -, le nombre d'élèves scolarisés dans des établissements privés d'Enseignement Secondaire était supérieur à celui des établissements publics.

3. La méthode grammaticale et pratique est une adaptation de la méthode traditionnelle aux nouvelles demandes des apprenants de langues modernes. Face à l'enseignement traditionnel, la méthode grammaticale et pratique envisage l'apprentissage d'une langue pour s'en servir dans les relations entre différents pays. Dans cette méthode, on introduit des objectifs pratiques, on inclut des activités orales - en s'occupant ainsi de la prononciation -, on limite l'exclusivité de la grammaire et on essaye d'améliorer la cohésion de l'enseignement du lexique. L'étude de la langue gagne des positions face à l'étude littéraire.

4. Le plan d'étude de 1845, connu comme Plan Pidal, régulait la création d'un Instituto de enseñanza media dans chaque province espagnole. L'enseignement de la langue française était réservé pour la troisième et la quatrième année du secondaire, d'abord comme matière optionnelle puis, à partir de 1857, comme matière obligatoire.

5. Pour les élèves qui n'avaient dans leur programme d'études aucune langue classique il était obligatoire de choisir les deux langues, anglais et allemand, en plus du français. C'est-à-dire que ces élèves étudiaient trois langues étrangères pendant leurs études secondaires.

6. Le manuscrit étant écrit avec une écriture très petite et serrée, nous avons eu des difficultés pour interpréter et transcrire certains mots et phrases employés par Nogués. Les mots illisibles sont remplacés par des points de suspension entre crochets; les coquilles, les calques et les hispanismes sont suivis du mot latin sic.

\section{RÉSUMÉS}

L'auteur présente un extrait d'un cahier utilisé pour la préparation de ses cours par un professeur de français qui a enseigné en Espagne de 1927 à 1971. Il s'était formé à l'Institución libre de Enseñanza de Barcelone qui a beaucoup contribué au renouvellement de l'enseignement des langues modernes. Et en effet, les textes élaborés par notre professeur, ancrés dans la réalité 
vécue par ses élèves, souvent pleins d'humour, reflètent les principes de l'école active - qui met au centre de l'action éducative l'enfant avec ses besoins et ses intérêts - et l'option méthodologique mixte, éclectique qui s'est manifestée un peu partout en Europe entre les deux guerres et qui a assoupli la méthode directe en faisant la synthèse des propositions "dures " venant d'Allemagne et des approches traditionnelles. Une option méthodologique très moderne pour l'époque en Espagne.

The author presents an extract from a notebook used for class preparation by a French teacher who taught in Spain from 1927 to 1971. He was trained at the Institución libre de Enseñanza in Barcelona, an institute that brought a significant contribution to the renewal of modern language teaching. And indeed, the texts drawn up by our teacher - lively and rooted in the lived experience of his students - reflect the principles of the active school, centering the educational experience around the students' needs and interests. But they also show the mixed or eclectic methodological approach to teaching that began to appear all across Europe between the two wars, and which brought flexibility to the direct method by combining the hardline propositions coming from Germany with more traditional approaches. A very modern methodological approach for the time in Spain.

\section{INDEX}

Mots-clés : Méthodologie, méthode active, méthode directe, approches traditionnelles, enseignement du français en Espagne

Keywords : Methodological approach, active school, direct method, traditional approaches, French teaching en Spain

\section{AUTEUR}

\section{MARÍA INMACULADA RIUS DALMAU}

Universitat Rovira i Virgili, Tarragona 